Dr. SaInz de AJA: In my opinion the reason the second patient did not improve, although he had sporotrichosis, is that he was syphilitic. Since he suffered from osteomyelitis, there was probably also some sequestrum. As is well known, these patients do not recover entirely until the sequestrums have been taken out.

\title{
NEW YORK DERMATOLOGICAL SOCIETY
}

Regular Meeting, Oct. 26, 1920

J. M. Winfield, M.D., President

PRURIGO. Presented by Dr. Whitehouse.

The patienit was presented merely as a matter of interest, on account of the rarity of the condition. It seemed to be a clearcut case of the old prurigo of Hebra. The patient, Anna M., aged 13 years, was born in this country. She had suffered from the disease since she was 17 months old. She presented a very definite case of Hebra's prurigo, on the arms, legs, body, and on the face. The papules, which at first appeared to be the color of the skin, later became inflammatory. The flexor surfaces were not involved. The axillary and inguinal adenopathy was very marked. Itching was intense. Treatment had been without avail; the only thing that gave any relief was baths of an antiseptic of the chlorin type. The lesions were more inflammatory than in some of the cases seen, but a notable point was the freedom of the flexor surfaces. The diagnosis was accepted without dissent.

\section{LYMPHOSARCOMA. Presented by Dr. Clarke.}

The patient, a police officer who had been shown before the Society in 1917, had a large lymphosarcoma, about the size of the palm, on his head. The case was referred by Dr. Eugene Poole, and at the time of presentation the members were unanimous in diagnosing the case as sarcoma. The patient was treated with a massive dose of roentgen-ray, resulting in a bad roentgen-ray burn of the second degree, but that cleared up and when presented he showed an excellent result of the treatment.

MELANOTIC SARCOMA TREATED BY ROENṬGEN-RAY. Presented by Dr. Cllarke.

The patient had been presented before the Society in the spring, after a massive dose of radium for melanotic sarcoma. At that time, she was presented to get the opinion of the members as to whether she should have further exposures or submit to surgical treatment. Some of the men had seemed to think that a portion of the malignant tumor still remained, but Dr. MacKee thought it was simply the radium burn. It was thought best to wait and see what happened. Finally, all the remaining black spots disappeared, leaving a frank roentgen-ray burn. The entire piece of tissue was excised and carefully examined, and the pathologist reported that nothing malignant was present. Then the lesion was skin-grafted. The patient was presented in May of last year. At present, there is no glandular enlargement anywhere. There was some keloid, but that seemed to be diminishing rather than increas- 
ing in size. Prior to the treatment, the lesion had existed for a long time. At first, it was about the size of a split pea, and then it began to increase rapidly until it was about the size of a half dollar.

\section{DISCUSSION}

Dr. Trimble said that he had seen the patient with the melanotic sarcoma before, and congratulated Dr. Clarke on the results. He had been wondering, however, whether the lesion would not have healed better without the skin graft.

Dr. Highman said that in reporting cases of this sort in which the histological diagnosis is not made, mention should be made of that fact.

Dr. Clarke said that he believed radium burns healed up more kindly than roentgen-ray burns. He had wanted to excise it on that account, for he wanted to get the whole area. It was minutely examined, for he wanted to see whether anything could be found, for Drs. Whitehouse and Fordyce believed that there was still some malignant disease remaining when the patient was presented before. He was not seeking a cosmetic effect at the time.

\section{RADIODERMATITIS. Presented by Dr. Howard Fox.}

R. C., a woman, aged 32 years, born in the United States, and a stenographer by occupation, six years before had consulted a physician in a neighboring city for an extensive hypertrichosis of the face. She stated that she had received from fifteen to eighteen roentgen-ray treatments in the course of three weeks. This was followed by a severe "burn with blisters," and at the end of a month the skin had peeled, leaving permanent disfiguration.

She now presented over the bearded region the results of a severe radiodermatitis, consisting of distinct pigmentation, telangiectasia and whitish superficial scars. No keratoses were present. The lesions were most distinct on the cheeks, chin and neck, the upper lip being only slightly affected. The patient was shown to emphasize the inadvisability of treating hypertrichosis with roentgen ray. The most careful treatment by measured doses has its dangers, while in this particular case it seemed probable that the operator lacked the necessary knowledge to use roentgen ray properly.

\section{DISCUSSION}

DR. WISE, replying to a query from Dr. Fox, said that he himself had used the Kromayer lamp in roentgen-ray telangiectasia but the results were very poor. He used radium in roentgen-ray keratoses with good results, but did not think one could accomplish very much with the Kromayer lamp in a case like this one as far as the vascular lesions were concerned.

Dr. Clarke said that he had had some success in cases of telangiectasia following roentgen-ray burns by the use of radium, and he recommended its application in this particular case. He would be rather skeptical of the effect of the Kromayer lamp in such a case. It could only be used with a blue filter and pressure and very prolonged exposure. The patient showed a tendency to freckle, and with a prolonged exposure the chances were that she would have freckles from it. He would treat such cases with the electric needle, and whenever possible radium. He thought the condition could be very much improved. Replying to a query of Dr. Wise, Dr. Clarke said that in many cases he had been able to make a dilated vessel close up with the needle. 
Dr. Highmsiif said that he did not see how it was possible for radium to produce that effect.

DR. Clarke said that he had been able to do that by filtering the radium with $0.75 \mathrm{~mm}$. to $1 \mathrm{~mm}$. of lead to get a very penetrating exposure.

Dr. Highman said that the vessels in nevi are neoplastic in their nature and represent a constantly proliferating process. For that reason they are influenced by radium, though not so much by the roentgen rays as by the beta rays. Telangiectasia produced by the roentgen rays has an entirely different origin. It is due to the fact that elastic tissue is destroyed, which eliminates vascular tone, so that the vessels naturally dilate under the increased pressure of the circulating blood, and being scarred vessels they do not respond to radium.

\section{LiChEN OBTUSUS. Presented by Dr. Highman.}

The patient, who was about 19 years of age, presented very curious lesions on the thigh, which began to appear six years before on the left thigh. Others developed later, and one appeared on the forearm. The itching had been very severe some years before, but had ceased. The lesions were regarded as warts. The patient had been carefully examined for lesions on the mucosa, but none were found. The treatment had consisted of mercuric chlorid, internally, and salicylated alcohol, externally.

\section{DISCUSSION}

DR. WhITEHOUSE said that it did not seem fair to criticize a diagnosis when one could not make a better one, but he could not subscribe to the diagnosis of lichen in this case. No doubt it was hypertrophic, but the lesions were so changed by the treatment that it was difficult to say what their nature was. It would be desirable to investigate the case by biopsy to see whether there was any resemblance to the conditions found in tuberculosis verrucosa. The isolated lesion on the back of the thigh that had not been touched looked like tuberculosis verrucosa.

Dr. Clarke said he could not make a diagnosis, but would like to know whether there was anything in the history to indicate that the patient had been taking bromid.

DR. Howard Fox said it was difficult to make a diagnosis in a case that had been so disguised by treatment. Some of the lesions suggested lymphangioma circumscriptum, and as had been said others suggested bromid lesions. It would seem that the diagnosis would have to be settled by the microscope.

DR. WISE said that he would accept the diagnosis as presented-lichen planus verrucosus.

Dr. Highman said that except for the lesion on the back of the thigh he questioned whether he would tonight have made the diagnosis submitted. When the patient was first seen, the lesions on the left thigh looked like lichen hypertrophicus and those on the abdomen were typical lichen papules. The other possibility that had been raised could be ruled out for two reasons: The boy had had the lesions for from four to six years, which would rule out a bromid eruption; and the fact that the lesions were not vesicular would rule out the possibility that Dr. Fox mentioned. Other possibilities-warts, different benign tumors, epithelioma-were not considered. He had wished to make a biopsy, but the boy was timid about it. The father, however, had consented to have it done, and a report would be submitted at the next meeting. 
LICHEN PLANUS OR NEVUS UNIUS LATERALIS? Presented by Dr. WISE for DR. FORDYCE.

A young woman, aged 26 years, presented lesions on the left arm and leg which were of fourteen years' duration. Slight itching was said to be present. The diagnosis seemed to lie between atrophic lichen planus and nevus linearis, but the biopsy did not show either. The lesions consisted of atrophic, small, pigmented papular lesions, arranged in linear fashion along the arm and leg.

\section{DISCUSSION}

DR. HIGHaAn did not know what to say about it. If it were lichen planus, it would be of the atrophic pigmented variety, but the atrophic variety is not usually pigmented. And if it was a nevus he did not know why it should itch and atrophy. He was at a loss to classify it.

Dr. TRIMBLE said he would not hesitate to make a diagnosis of nevus in the case. The fact that there were some flat papules would not change his opinion.

Dr. Howard Fox said that he had a patient under treatment with a warty linear lesion extending down the back of the thigh from the buttock. As it had existed from birth, he felt sure that it was a nevus in spite of the fact that the patient had complained a good deal of itching.

DR. CLARKE said he could not reconcile a case of linear nevus with such extreme itching, the atrophy and the rather diffuse distribution. He was inclined to a diagnosis of atrophic lichen planus.

DR. WISE said that a biopsy report would be submitted at the next meeting.

\section{VERRUCA. Presented by Dr. Trimble.}

The patient, a Porto Rican, aged 36 years, with a negative family and personal history, presented a condition which began on the dorsum of the left foot twenty-five years before as small verrucous lesions. At that time, the patient was working barefooted in the tobacco and sugar fields of Porto Rico. The lesions gradually enlarged in size, and at the end of six months spread to the dorsum of the right foot, and had since continued to enlarge and spread until they attained the extent exhibited. A few years before, after scratching the feet and getting blood on the fingers, the patient noticed lesions appearing on the fingers.

The condition presented consisted of discrete and confluent brownish verrucous lesions, varying in size from that of a small pea to several inches in diameter, covering the feet and stretching up the leg, and also appearing on the fingers. The Wassermann reaction was negative. The pathologic report was verruca.

\section{DISCUSSION}

Dr. WhITEHOUSE agreed with the diagnosis.

Dr. Clarke said that he had seen the patient before he received any treatment, and agreed with the diagnosis. The lesions when treated with salicylic acid applications had improved more than those treated with roentgen ray. He expressed doubt whether a good result could be obtained with the lesions unless they were cleared of the crusts or scales before the roentgen-ray treatment was applied. 
Dr. Winfield agreed with Dr. Clarke that the foot that received the salicylic acid looked better, and also agreed that if the lesions were first curetted and then the roentgen-ray applied a cure would probably result.

Dr. TRIMBLE said that the lesions had received no treatment prior to being treated with the roentgen ray. They were not curetted, but were soft when they were irradiated.

\section{LESIONS RESULTING FROM MORPHIN HYPODERMICS. Presented by Dr. Wise for Dr. Fordyce.}

A young woman, aged 27 , who for six years had been addicted to the morphin habit, presented lesions which covered her arms where the injections had been made. There were the characteristic hard bluish masses, a half centimeter in diameter, some discharging pus and serum.

\section{RECURRENT (STREPTOCOCCIC) DERMATITIS OF FACE. Presented by Dr. Howard Fox.}

W. H. K., a man 41 years of age, born in the United States, an administrative assistant in the U. S. Public Health Service, six months before had suffered from an eruption similar to the present one, lasting five days. A second attack, lasting seven days, occurred three months later. The present attack appeared five days before, and was now rapidly subsiding. When seen on the second day, he presented a dermatitis about the eyes, nose, and cheeks, more severe on the left side. The affected area was red, hot and edematous, but showed no vesiculation or sharp demarcation characteristic of erysipelas. In this, as in previous attacks, he complained of slight constitutional symptoms consisting of anorexia and headache. There was also a slight rise of temperature. He complained of a burning sensation of the skin but no itching. He had suffered from a severe attack of poison ivy dermatitis six years before. Previous to each of these attacks, he had not been exposed to his knowledge to any cutaneous irritant. He had suffered a good deal from nasal catarrh and from indigestion. Examination of the nose revealed no gross lesions. Examination of the swabs from the nasal passages, after irrigation with sterile saline, were made at the Marine Hospital (Stapleton). The result was as follows: "On Loeffler's blood serum a profuse growth within twenty huurs, which on smear preparation showed a gram-positive micrococcus. On transplanting to blood dextrose broth, a hanging drop showed a micrococcus with no definite morphology similar to that of streptococcus. Culturally, organism isolated was Micrococcus aureus of low virulence."

\section{DISCUSSION}

Dr. Highman said the thing to do in such a case was to get the serum from the lesions during an acute attack, make a vaccine and treat the patient.

$D_{R}$. WISE said that he had very little personal experience in such cases, but that he had seen patients treated by others, and that the procedure outlined was the same as that referred to by Dr. Highman.

Dr. Fox replied that nothing abnormal had been found by the rhinologist at the Marine Hospital. Dr. Fox had recently presented before another society a patient who suffered from a permanent elephantiasic swelling below the eyes. An attempt had been made by one of the Public Health surgeons. to obtain fluid for culture by puncturing the swollen tissue, but without success. 
Dr. Winfield said he had seen several cases of this condition and had always found some lesion about the nose which seemed to light up the attack. He believed the stock vaccines would do as well as the autogenous vaccines.

Dr. Fox replied that at the hospital they stated there was no visible lesion. At the Academy Section last winter he had presented a man with frequently recurring edema below the eye, and some of the physicians in the Public Health Service tried this procedure but were unable to get enough fluid to make a culture.

EPIDERMOLYSIS BULlosA. Presented by Dr. Wise for Dr. Fordyce.

A woman, aged 27 years, had had the present condition since last March. She was married and had one child. For the last eight months she had had lesions similar to those exhibited. The lesions consisted of scattered vesicles and small bullae on the face, neck and hands. They often arose on striking the skin by accident or otherwise. It seemed to be a case of epidermolysis bullosa of the nonhereditary type. No other members of the family showed any similar lesions. The patient also presented little miliary epidermal cysts on the shells of her ears and on the fingers. The outer surface of the elbow regions exhibited the characteristic atrophic scarring, resulting from previous lesions.

DISCUSSION

Dr. Highman said that he had not a clear perception of the case. It would seem that if it were epidermolysis it would be possible to elicit Nikolsky's sign. The lesions on the ear looked like ordinary dermatitis in that location. He did not understand what was meant by the term epidermolysis bullosa acquisita.

PARAPSORIASIS GUTTATA. Presented by Dr. Wise for Dr. Fordyce.

A boy, aged 12 years, born in Russia, had been troubled with the lesions for twelve months. He had been presented before the Academy of Medicine, at which time he showed a great number of small, pinkish macules, isolated and confluent, some of them slightly scaly. He had had a considerable number of treatments, but according to his parents they had had absolutely no effect on the condition. The impression was that he had psoriasis. Later, on account of the type of the eruption, and its peculiar resistance to treatment, a diagnosis of parapsoriasis guttata was made.

\section{DISCUSSION}

DR. Fox and DR. Trimble agreed with the diagnosis.

DR. WISE said that he had treated a patient referred by Dr. Wallhauser with roentgen rays and the eruption cleared up entirely. While the patient was under treatment, however, a new crop of the lesions appeared in the interval between two weekly applications of the rays.

Dr. High man said that in one of the issues of a German journal a physician had related his experience with parapsoriasis and purports to have cured all his patients by injections of one-tenth grain of pilocarpin every other day. The patients improved after two or three injections and were cured in a few weeks. 


\section{ACRODERMATITIS CHRONICA ATROPHICANS WITH FIBROTIC \\ TUMORS. Presented by Dr. WISE for DR. FordyCE.}

A married woman, aged 45 years, born in Russia, had had the lesions presented for ten years. The history given was rather vague, but it was gathered that the trouble began with a feeling of coldness in the feet and the skin became "hard." The trouble had traveled up toward the groin. She also presented distinct fibrotic nodules associated with atrophy on both elbowsthe so-called ulnar band-and the characteristic cutaneous atrophy of both legs.

The diagnosis was accepted without dissent.

\section{DISCUSSION}

Dr. Highman said that if therapeutic observations were of any interest, he could tell of a similar case with band-like lesions, and on the homeopathic principle of similia similibus curanter he had used the roentgen rays which was followed by a very rapid limbering of the tissue. Whether or not that was simply a coincidence he could not say.

PHENOLPHTHALEIN ERUPTION. Presented by Dr. Wise for $D_{R}$. FORDYCE.

E. A., a married woman, aged 25 years, presented herself at the Vanderbilt Clinic with a fresh outbreak of an eruption which had recurred, at intervals of from one to four weeks, during the past three years. The forehead, face, shoulders, axillae, arms and thighs, showed a number of macular and urticarial lesions from 1 to $3 \mathrm{~cm}$. in diameter, bluish red, and suggesting erythema perstans. Several days later, when the eruption had subsided, she was given two 5-grain tablets of phenolphthalein, and the following day many of the spots had appeared in all their intensity.

\section{DISCUSSION}

Dr. Howard Fox said that phenolphthalein seemed to produce an eruption that was more or less characteristic. This consisted of well defined, round or oval erythematous or urticaria-like coin-sized lesions, followed by rather persistent pigmentation. He had had a similar case due to an ingestion of exlax (a patent preparation containing phenolphthalein).

DR. WINFIELd said that he had seen a somewhat similar eruption from antipyrin. From this coal tar preparation, however, the patches were more erythematous and not purpuric. The erythematous patches often left a brown stain which was more or less chronic.

LICHEN PLANUS. Presented by Dr. Wise for Dr. Fordyce.

G. V., a sturdy fireman, aged 28 years, presented the typical lesions of lichen planus on his lips and chin, and on the backs of his hands. The noteworthy point was the extensive involvement of the vermilion portion of the lips and the complete absence of lesions on the mucous surface of the lips and interior of the mouth. The lesions on the lips had been present for seven weeks; those on the hands for one week. 
CASE FOR DIAGNOSIS (PAPUlONECROTIC TUBERCUlid?), Presented by Dr. WISE for DR. FordyCE.

H. R., aged 57 years, a salesman of Russian birth, had lived in America eight years, and gave a negative family history. His face, scalp and extremities bore closely crowded lesions in various stages of development, ránging from $1 / 8$ to $1 / 4$ inch in diameter. There were many violaceous papules, more or less firm to the touch, some capped with crusts whose removal disclosed the presence of dry, necrotic centers. Many older lesions, dry, atrophic and pitted, were interspersed with these. The lesions on the face resembled acnitis. The patient said the eruption had been present only for the last fifteen months. His health was good otherwise, but he complained of severe pruritus of the affected parts. A biopsy was obtained, but the histologic examination did not show it to be papulonecrotic tuberculid.

\section{DISCUSSION}

Dr. Highman said it was either papulonecrotic tuberculid or prurigo, and he could not see any diagnostic evidence of the former.

Dr. Howard Fox said the important point to decide was whether the condition was an inflammation or a granuloma. The evidences of intense itching and the absence of pitted scars (as in a papulonecrotic granuloma) spoke strongly in favor of an inflammatory condition. It was of less importance to decide whether the disease should be classed as a prurigo or a dermatitis herpetiformis.

Dr. Whitehouse agreed with Dr. Fox that the lesions on the forehead were not scar-forming lesions. In consequence of the intense itching and grouping of lesions, it impressed him as a peculiar type of Duhring's disease, or some kind of inflammatory disease.

Dr. Wise, replying to a query from Dr. Lane, said that he did not think the lesions on the forehead itched as did those on the hands. He would submit a biopsy report at the next meeting.

Dr. WINFIELD said that he saw nothing in the case to make him think of dermatitis herpetiformis.

\section{UNUSUAL TYPE OF SYPHILODERM : REPORT OF CASE. Presented}

by Dr. HighMan.

A man, aged 50 years, had a pruriginous annular group of granulomatous lesions on the scrotum and elsewhere. There were about eight lesions, ring shaped, plaque shaped or papular, deeply set in the subcutis, varying in size from 0.25 to $2 \mathrm{~cm}$. in diameter, violaceous and of a hard but elastic consistency. Clinically, they suggested granuloma, a diagnosis substantiated microscopically, and made despite the localization. Severe itching was complained of. Syphilis was denied.

Under roentgen-ray treatment no improvement was evident, and the question of syphilis was again raised. Although the Wassermann test was negative, antispecific treatment was begun, and within forty-eight hours after the first arsphenamin injection, the lesions had shrunk to one-half their original volume, the itching having stopped at once.

In this connection it must be added that the right index finger was swollen. Radiographic examination showed the bones to be normal. Thus the finger con- 
dition represented an infiltrated edema of the soft parts. This, too, healed under specific treatment.

The patient was shown because of these points: (1) itching relieved by antisyphilitic treatment, (2) the rare character of the lesions, and (3) the weak serum reaction.

\section{SKIN SHEDDING (KERATOLYSIS EXFOLIATIVA CONGENITA). REPORT OF CASE. Presented by Dr. Howard Fox.}

Mrs. L. E. G., aged 28 years, born in Texas, was seen in consultation eight months before. She stated that during her entire life she had suffered from peeling of the skin in flakes similar to those of scarlet fever. The desquamation affected the entire body and was never associated with redness or any subjective symptoms. At intervals of a week or less the skin shedding would become active. No one else in her family had suffered from a similar condition. She had always enjoyed good health and had no theory to offer regarding the cause of her affection. The desquamation was not affected by the menstrual periods and did not vary during the different seasons of the year. It was not affected by the use of oily applications. Her chief complaint was the embarrassment caused by the appearance of the skin at times.

On examination she showed a slight, flaky desquamation of the chest of a scarlatiniform type. There were no inflammatory changes, with the exception of a definite factitious urticaria. There was no evidence of ichthyosis, her skin in general being remarkably soft and smooth. There were no abnormalities of the hair and nails. She was well nourished and appeared to be in the best of health living an outdoor life of leisure.

\section{DERMATITIS VENENATA FROM ORIENTAL CASHEW NUT. REPORT OF CASE. Presented by Dr. Howard Fox.}

Miss M. L., aged 32 years, was a laboratory worker in the U. S. Department of Agriculture. She was born in the United States, but until lately had lived in Germany for fifteen years. During the last four months she had been working in the laboratory where she was constantly handling various plants. She was first seen on September 11, when she presented a severe generalized dermatitis similar to that from rhus poisoning. This was most marked on the face and upper extremities and consisted of a profuse vesicular and itchy eruption, assorated with extreme edema, the eyes being closed and the features unrecognizable. The patient was confined to her bed. The eruption gradually subsided but the edema did not disappear when treated with cold compresses or boracic acid as fast as is usually noted in rhus dermatitis. At the end of three weeks, the eruption had disappeared except for flaky desquamation of the hands. This was entirely well about two weeks later.

The eruption appeared on the day after she had handled specimens of Oriental Cashew nut (Anacardium orientale), the fruit of a tall tree of India. While she was cutting several nuts and making microscopic sections for about an hour and a half, some of the juice of the mesocarp came in contact with her hands, staining them a blackish hue. Two other workers, a man and a woman in the same laboratory, handled the same nuts and on the following day presented an eruption on the face and hands. The man, who had handled the specimens longer than the woman, and whose hands had become slightly blackened, was said to have had a more severe eruption. One other laboratory assistant had touched the nuts without ill effect, and the same was true of the helper who washed the instruments which had been used in making sections. 
As a possible cause of the dermatitis, it should be said that the patient had been in the Catskill Mountains five days before the eruption appeared. She did not, however, handle any poison ivy leaves. She had never previously suffered from rhus poisoning to her knowledge, though it must be said that during the greater part of her adult life she had lived in Germany.

The patient appeared to be susceptible to irritation from plants, as on a previous occasion (end of June) she had handled sanguinaris powder and on the following day an eruption had appeared on the face, neck and chest, and between the fingers. It was red and showed "blisters," and lasted ten days. This attack also followed a short walk in the country (three days previously) though she did not, as far as she was aware, come in contact with poison ivy. Very recently she had again suffered from a slight eruption on the wrists, on the day after she had handled pistachio nuts. This lasted only two days. On this occasion she had immediately used alcohol and zinc oxid ointment.

The oriental cashew nut, which was presumably the cause of the dermatitis in this case, is to be distinguished from the cashew nut which is a native of tropical America (Anacardium occidentale). The oriental, like the native species, is known to be a cutaneous irritant.

\section{DISCUSSION}

Dr. WISE inquired concerning the five days' incubation period of poison ivy.

Dr. Fox replied that the eruption came on very suddenly after the exposure. He had never seen a case in which the incubation period lasted five days.

DR. Winfield said that he had had personal experience. On some occasions the eruption did not develop for ten days to two weeks after exposure, although there was considerable itching before the eruption appeared.

Dr. WISE said that he had seen a case in a clinic on a Monday in a man who stated that he had been in the woods on the preceding day, Sunday, that is. sever days previously.

Dr. Highman told of a patient seen on a Wednesday, the exposure having occurred on the previous Sunday, or four days earlier.

\section{PHILADELPHIA DERMATOLOGICAL SOCIETY}

Regular Monthly Meeting, Nov. 8, 1920

Milton B. Hartzel., M.D., Presiding

\section{MYCOSIS FUNGOIDES. Presented by Dr. Hartzell.}

A printer, 50 years old, had a universal dermatitis for over two years. In most situations it exhibited a smooth, shiny red infiltration, itchy and slightly scaly, the latter condition being most marked on the palms. Significant features of the disease were numerous eruptions on the trunk in the form of scores of flat, infiltrated, slightly elevated, scaly areas ranging from the size of a pea to that of a fingernail. These tumors had been present for the past four months. They were most abundant on the upper trunk, both anteriorly and posteriorly. Adenopathy was marked; the inguinal and axillary glands stood out in relief. No laboratory findings were reported. A moderate degree of conjunctivitis was present. 